\title{
Decreasing Energy Level in Living Organisms
}

\author{
Vedant Khandelwal \\ (ECE, UPTU, India)
}

\begin{abstract}
Here in this article, the reader is made to think again on the universal saying, "Energy is constant in this universe." In the end of the article the reader will think twice about the energy content in a living organism. As it is said that the total energy in this universe is constant, at the end of the article the reader will try to think that the energy in living organisms is decreasing with time!!!
\end{abstract}

Keywords: Energy, Life.

\section{Introduction}

A few days ago, I read a written form of essay, delivered by John Dobson as a lecture at the Vedanta Society, Berkeley, USA, on 12th October 1980.

It was titled as "Einstein's Physics of Illusion", but had nothing to do with "magic or wizards" or "illusion related to optics". It was a bit related to the total energy in the universe.

This is what gave birth to an idea in my mind to write down about this.

\section{Headings}

As the title tells you, this article is about the decreasing energy level in living organisms.

I will be discussing this in several parts:

a) Total energy in universe.

b) Energy within living organisms.

c) Rising population.

d) Conclusion

\section{a) Total Energy In Universe:}

As we all know, and as it is a universal fact that "total energy in the universe is constant", it means that the total amount of energy in the whole universe, remains constant. It just changes its form. Energy is broadly classified as- gravitational energy, kinetic energy, radiation, electricity, magnetism and nuclear energy. Many of the scientists and researchers have shown that the universe is based on two basic energies- Gravitational energy and Potential Energy. Many more forms are there but basically the idea is that the energy keeps changing its form. For example- the chemical energy of fuel turns into heat and mechanical energy of the engine!!

This change goes for each and everything in this universe. It could be from your cell phone to your highly digitalized automatic sports car, from your CFL to your air-conditioner and whatever you see around you!

This was all about the theory or fact which we have been learning and studying from our childhood, but what if there is change in energy? What if the energy, the total energy in the universe is increasing or decreasing? What if there is a considerable change in the amount of energy in the universe but as the size of universe is huge; we might not have noticed it!

I know I may be sounding insane as many scientists and researchers have already given their theory for the amount of energy being constant in this universe, but what if you have a sense of confusion about this after reading this whole article? What if you start thinking that there is a chance of energy to keep decreasing or increasing but not being constant?

My motto is not to create confusion or ambiguity in your mind, but to know whether my thinking is wrong or right.

So, let us move towards the next part of our article.

\section{b) Energy Within Living Organisms:}

We have been taught this in our primary classes that we need energy to do work or plants need energy to make their own food! Or even in very simpler terms, a home-made example, Mummy always says to her child, "Finish the glass of milk and then you'll have a lot of energy to be strong!"

this all examples, daily life examples tells us that each one of us(not just human beings, but all living organisms) have some energy within us!

Now, coming towards the scientific part of this topic-

LIFE! What is it? A science topic or a spiritual topic! An energy form or just a process! 
According to Wikipedia- "It is a challenge for scientists and philosophers to define life in unequivocal terms. This is difficult partly because life is a process, not a pure substance. Any definition must be sufficiently broad to encompass all life with which we are familiar, and must be sufficiently general to include life that may be fundamentally different from life on Earth. Some may even consider that life is not real at all, but a concept instead."

Tiny energy factories are there within plant's and animal's cells, which keep on transforming energy which is necessary for life! In plants, we call those tiny factories as chloroplasts. Energy from the sun is collected by them and by photosynthesis they convert carbon dioxide and water into sugar molecules. Mitochondria, the tiny energy factory of animals transform this sugar molecule from the plant into a versatile energy currency in the form of adenosine triphosphate (ATP). This molecule stores the energy, required for each and everything our body does.

"Sun is the primary source of energy." this is the line which each and every one of you may have read once. Combining the two facts, we get, sun is the only source of energy and the total energy in the universe is constant.

So, we may get an idea from the above statement that if Sun's providing energy continuously and the total energy is constant, the energy received by us must be transforming and going somewhere in this universe. But, what if the number of recipients increases? The only source is Sun, the amount of energy reaching energy by $10 \%$ rule is somewhat constant but what if the number of persons (energy receivers) increase rapidly? This makes us move to our next part.

\section{c) Rising Population:}

Rising population is a big cause of increasing dangers in our ecosystem. Be it pollution or deforestation, the basic cause is rapid rise in population. Here, in my article, rising population is the key part which led me to think to this level.

Let us understand this mathematically, math-haters don't be disheartened, we'll be using just the simple mathematics for this.

Let the energy reaching the earth surface is $=\mathrm{x}$ (not taking the units)

Let the number of people on Earth in year $2001=y$

Let there be number of plants on Earth in year $2001=\mathrm{z}$

We know that, " $y$ " depends on " $z$ " and " $z$ " depends on " $\mathrm{x}$ ".

Now, after $10 \mathrm{yrs}$, in the new census, the number of people increased dramatically and hence the number of plants decreased at a high rate due to pollution and urbanization. Till now (2014), the records might have been gone to more extremes.

So, in today's date,

$\mathrm{x}$ is still the same.

$\mathrm{y}$ has increased largely.

$\mathrm{z}$ has decreased largely.

But according to the theory that "animals live on plants and plants live on sun's energy", when the number of plants decreased, the number of animals (including human beings) must decrease as they were directly proportional.

But what we see is that keeping the sun's energy received by Earth at a constant value, the rate of animal population is still increasing with decrease in number of plants on Earth. This makes us to reach to the conclusion part of my article.

\section{Conclusion}

We saw in the previous part that the number of animals (including human beings) is increasing. Again using some mathematics:

If the total amount of energy transformed by one person in a fixed time interval is $=\mathrm{m}$

So, the total amount of energy transformed by five persons within the same fixed time interval will be $=5 \mathrm{~m}$ This shows the energy requirement increases with increase in population!

But, again coming to the same thing, we know that the primary energy producer is producing the same amount of energy or rather I should say that the amount of energy reaching Earth is same. If the population (number of energy receivers) keeps on increasing, the total energy consumption required will be more and more, which is not possible! So, the possible conclusion might be that, the amount of energy consumption by animals is decreasing with increase in population. 
Dear reader, Is this true? Is the amount of energy transformation or energy consumption decreasing with increase in population? Are we adapting to this situation without noticing it? Is it a matter of subject to be thought upon? As I said in the starting, are you now confused that whether the energy is constant or is it changing? And if it is constant, is the amount of energy consumption decreasing? What's your conclusion?

\section{References}

[1]. http://www.quanta-gaia.org/dobson/PhysicsOffllusion.html

[2]. http://en.wikipedia.org/wiki/Life

[3]. http://hyperphysics.phy-astr.gsu.edu/hbase/biology/enercyc.html 